\title{
Spontaneous closure of coronary artery fistula
}

\author{
DAVID HACKETT, K A HALLIDIE-SMITH \\ From the Department of Clinical Cardiology, Royal Postgraduate Medical School, Hammersmith Hospital, London
}

SUMMARY A coronary artery fistula diagnosed in a 1 year old girl closed spontaneously during childhood. This outcome has been documented in only three previous cases.

The natural history and management of coronary arterial fistulae remain incompletely defined, particularly with respect to the role and timing of surgery in asymptomatic patients. ${ }^{1-3} \mathrm{We}$ report a case of a right coronary artery to right ventricle fistula which underwent spontaneous closure. This outcome has been only rarely reported. ${ }^{4-7}$

\section{Case report}

A West Indian girl was referred in 1976 at the age of 1 year for investigation of an asymptomatic murmur, which had been discovered on a routine infant examination. Her medical history, birth and development, was unremarkable, and no murmurs had previously been noted. Examination at that time detected bounding aterial pulses and a systolic thrill with a grade $4 / 6$ continuous murmur at the lower right sternal edge. The clinical diagnosis, based on the character and site of the murmur, was a coronary fistula. An electrocardiogram showed changes of biventricular hypertrophy, and a chest radiograph indicated minimal cardiomegaly and pulmonary plethora. Cardiac catheterisation in January 1977 (aged 11/2 years) confirmed a left to right shunt at the right ventricular level with a calculated pulmonary to systemic flow ratio of 1.4 . Haemodynamic pressures in the right atrium, right ventricle, pulmonary artery, left ventricle, and aorta were all normal. Angiography of the aortic root showed a dilated proximal right coronary artery with a fistulous communication to the right ventricle just below the outflow tract (Figure). It was decided to manage this conservatively, and advice regarding prophylaxis against infective endocarditis was given.

During the period February 1977 to February 1979 the patient's mother reported that the systolic thrill had disappeared. The patient remained asymptoma-

Requests for reprints to Dr D Hackett, Department of Clinical Cardiology, Royal Postgraduate Medical School, Hammersmith Hospital, Du Cane Road, London W12 0HS. tic. At the end of this period, when aged 4 years, she had no evidence of bounding pulses, systolic thrill, or any continuous murmur. Both the electrocardiogram and chest radiograph were normal. A treadmill exercise test (Bruce protocol) was negative for symptoms or electrocardiographic changes. Repeat cardiac catheterisation in August 1983 (at 8 years) again showed normal haemodynamic pressures, but no evidence of any left to right shunting could be detected. Angiography again showed a dilated right coronary artery ostium but no fistula was evident. A small right coronary artery distal to the previous fistula site was found (Figure).

\section{Discussion}

Congenital coronary arterial fistulae, first reported in 1865, were considered rare curiosities before the advent of angiography and selective coronary arteriography. ${ }^{8}$ In recent years, however, increasing numbers of cases have been reported, and almost 300 have now been published. ${ }^{3}$ The anatomical and clinical variations and haemodynamic profiles have been well reviewed elsewhere. ${ }^{2}$

Principles of long term management of coronary arterial fistula have not been clearly defined. The natural history is poorly understood, and the proportion of symptomatic patients varies in different series. ${ }^{1-3}$ Nevertheless, symptoms do appear to relate to increasing age. Futhermore, the incidence of fistula related complications, such as congestive heart failure, myocardial ischaemia/infarction, and death, appears to increase with older age groups. ${ }^{1}$ In contrast the risk of infective endocarditis seems constant at $3-4 \%$ of patients with coronary arterial fistula. For these reasons routine early elective closure of the fistula has been recommended. ${ }^{1-39}$ Previous reports of surgical treatment have resulted in an appreciable mortality (4\%) and morbidity (10-15\%), the latter mainly being due to recurrence of the fistula and to 

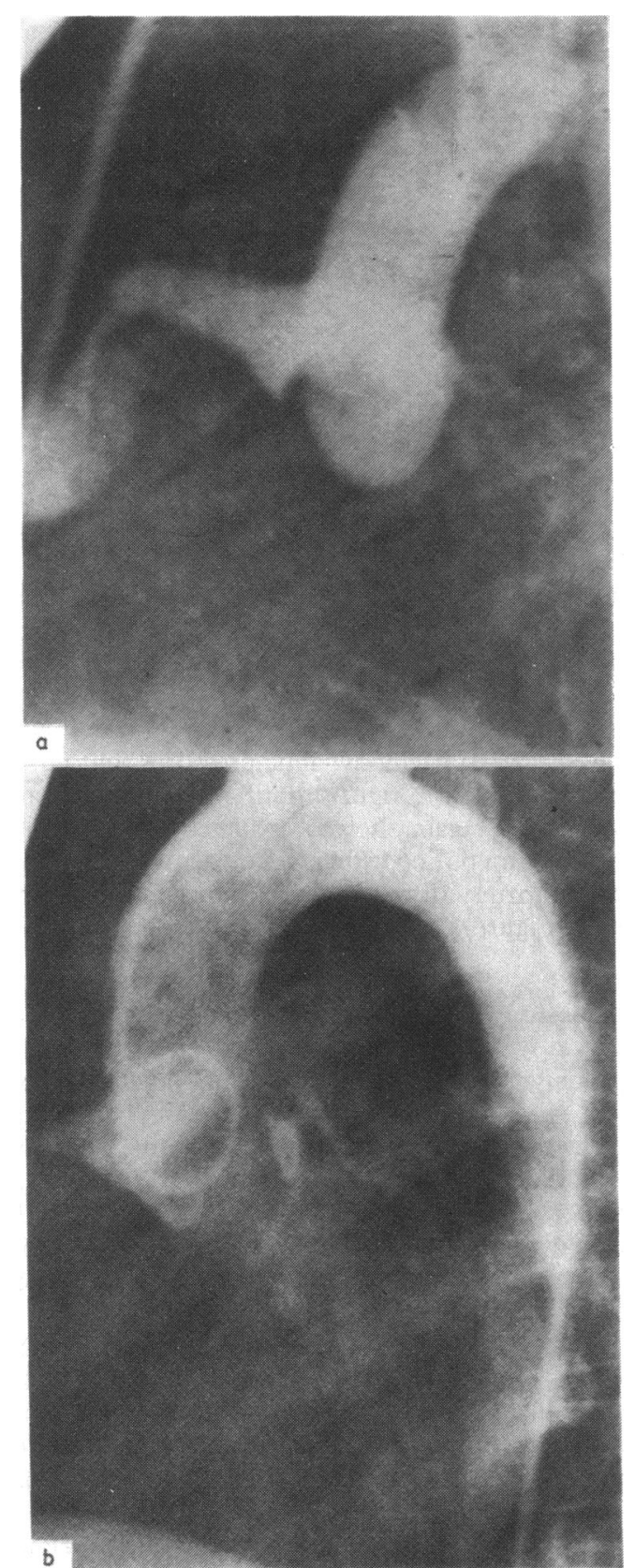

Figure Angiograms of the aortic root in a child at (a) aged 11/2 years showing a fistula from the right coronary artery to the right ventricle and (b) at age 8 years showing closure of the fistula.

myocardial ischaemia and infarction. ${ }^{2}$ With the availability of the cardiopulmonary bypass technique and since the introduction of coronary artery bypass surgery recent series have emphasised the absence of any operative related mortality and a low long term morbidity (3-5\%). ${ }^{39}$

Spontaneous closure of coronary arterial fistula has been angiographically documented in only three previously reported cases. All were children $(4,5$, and 14 years old), and all had fistulae terminating in the right ventricle. 457 In another case, a continuous murmur from a confirmed coronary artery fistula terminating in the right ventricle disappeared at the age of 4 years, but the presumed fistula closure was not confirmed angiographically. ${ }^{6}$ Jaffe et al have reported a case of coronary arterial fistula in a 29 year old woman whose continuous murmur had disappeared by the age of 44 years, but this was found to be a result of atherosclerotic occlusion of the right coronary artery. ${ }^{10}$ Our case is the fourth reported to have undergone documented spontaneous closure, the mechanisms of which remain obscure. It has been postulated that a high flow leads to shear induced endothelial damage leading to premature atherosclerosis and thrombosis. ${ }^{10}$ The right coronary artery distal to the occluded fistula is small and irregular (Figure) and indeed may give rise to future ischaemia. Thrombosis could also occur independently in the intramyocardial and endocardial venous sinusoids. All the reports of spontaneous closure of coronary arterial fistula have been in children, and all such fistulae have terminated in the right ventricle. Thus a further possible mechanism of spontaneous closure, similar to that occurring in muscular ventricular septal defects in children, is by presumed fibrosis and local myocardial hypertrophy.

Routine elective surgical closure of coronary arterial fistula can be recommended in adults, either on the basis of symptoms or to prevent future complications. Surgical closure in symptomatic children is also indicated. ${ }^{1}$ The role of surgery in asymptomatic children with coronary arterial fistula remains to be defined, both because of the uncertain natural history and the (small) possibility of spontaneous closure.

\section{References}

1 Liberthson RR, Sagar K, Berkoben JP, Weintraub RM, Levine FH. Congenital coronary arteriovenous fistula. Report of 13 patients, review of the literature and delineation of management. Circulation 1979; 59: 849-54.

2 Rittenhouse EA, Doty DB, Ehrenhaft JL. Congenital coronary artery-cardiac chamber fistula. Review of operative management. Ann Thoracic Surg 1975; 20: 468-85.

3 Lowe JE, Oldham HN Jr, Sabiston DC Jr. Surgical management of congenital coronary artery fistulas. Ann Surg 1981; 194: 373-80.

4 Shubrooks ST, Naggar CZ. Spontaneous near closure of 
coronary artery fistula. Circulation 1978; 57: $197-9$.

5 Mahoney LT, Schieken RM, Lauer RM. Spontaneous closure of a coronary artery fistula in childhood. Paediatr Cardiol 1982; 2: 311-2.

6 Morgan JR, Forker AD, O'Sullivan MJ Jr, Fosburg RG. Coronary artery fistulas: seven cases with unusual features. Am f Cardiol 1972; 30: 432-6.

7 Griffiths SY, Ellis K, Harlof AJ, et al. Spontaneous complete closure of a congenital coronary artery fistula. $\mathcal{f} \mathrm{Am}$ Coll Cardiol 1983; 2: 1169-73.
8 Krause $W$. Uber den Ursprung einer akzesserischem a. coronary aus der a. pulmonalis. Zeitschrift fir rationelle Medizin 1865; 24: 225.

9 Urrutia-S CO, Falaschi G, Ott DA, Cooley DA. Surgical management of 56 patients with congenital coronary artery fistulas. Ann Thoracic Surg 1983; 35: 300-7.

10 Jaffe RB, Glancy DL, Epstein SE, Brown BG, Morrow AG. Coronary arterial-right heart fistulae: long term observations in seven patients. Circulation 1973; 47: 133-43. 